\title{
EDITORIAL
}

\section{La vitamina reina o la reina de las vitaminas}

$\mathrm{L}$ a vitamina D clásicamente es reconocida como el regulador del metabolismo del calcio y del fósforo. Por ejemplo, el raquitismo y toda una serie de alteraciones musculares relacionadas, no tenían una etiología conocida hasta que se logró la identificación en 1922 del secoesteroide que hoy conocemos como vitamina D.

El interés en la vitamina fue creciendo en los años siguientes cuando se descubrió su vía de activación a 25-hidroxivitamina en el hígado y su producto activo, la 1,25 hidroxivitamina D en el riñón.

Su investigación ha continuado en relación con sus metabolitos, actuando como agente terapéutico en diferentes enfermedades metabólicas, lo que ha llevado a poder comprender su fisiología como hormona pleiotrópica con acción en tejidos humanos.

La vitamina D es una vitamina liposoluble. Cuando es originada en las plantas, es conocida como ergocalciferol (vitamina D2) y la forma animal se conoce como colecalciferol (vitamina D3). La fuente de la vitamina D2 para los humanos se encuentra en ciertos alimentos, mientras que la D3 puede derivarse de su síntesis endógena como también de la dieta. La vitamina D3 es sintetizada en la piel por influjo de los rayos ultravioleta a partir del 7- dehidrocolesterol.

Más tarde, tanto la D2 como la D3 son hidroxiladas en la posición 25 en el hígado, dando origen a la 25-dihidroxivitamina $\mathrm{D}$, que representa la mayor parte circulante, la cual al final es activada por medio de una hidroxilación en la posición 1 en el riñón, dando origen a la 1,25 dihidroxivitamina D. La enzima responsable de la formación de la 1,25 hidroxivitamina D en el riñón, llamada CYP27B1, se encuentra igualmente en varios tejidos extrarrenales, pudiendo llegar a producir 1,25 dihidroxivitamina D de una forma para o autocrina.

Los investigadores Mellanby y McCollum, el primero inglés y el segundo norteamericano, trataron a ratas que padecían raquitismo con aceite de hígado de bacalao, que lograron recuperarse de esta enfermedad. Al publicar sus hallazgos en 1922, McCollum, siguiendo el orden alfabético de las vitaminas, y habiendo ya sido asignadas las letras para las vitaminas A y B, llamó a este nuevo milagro la vitamina D.

En la década de los sesenta, el grupo de Héctor F. DeLuca esclareció el metabolismo de estas sustancias en el organismo, bien por vía de la irradiación solar de la piel y activación de la provitamina D o por absorción intestinal de calciferoles, hidroxilándose estos preocursores en el hígado y luego en el riñón para dar lugar al calcitriol, hormona final con actividad metabólica. Las mediciones de la 25-hidroxivitamina D se aceptan actualmente como el mejor índice de los depósitos de vitamina D. En la circulación, la 25-hidroxivitamina D se une a su proteína transportadora DBP, lo que le confiere características similares a una hormona ${ }^{(1)}$.

Su deficiencia es un problema de salud pública a nivel internacional. Según estadísticas de los Estados Unidos, las concentraciones bajas de 25-hidroxivitamina D tienen un rango de $27 \%$ a $91 \%$; lo mismo ocurre, de forma paradójica, en los países tropicales como Colombia ${ }^{(2-6)}$, en donde los estudios que existen muestran déficit de esta sustancia, a pesar de que en algunas ocasiones existe la dificultad de contar con laboratorios que posean los recursos tecnológicos y personal capacitado para realizar su dosificación.

Las manifestaciones extraesqueléticas relacionadas con su déficit incluyen trastornos dermatológicos y cardiovasculares, enfermedades malignas y desórdenes metabólicos.

En el año 2014, con motivo del XXIII Congreso Colombiano de Medicina Interna en Cartagena, se presentó la revisión de la literatura médica aparecida entre enero de 1970 y noviembre del 2004 en las bases PubMed, Embase y Web of Science, identificando las publicaciones internacionales relacionadas, buscando la asociación con la latitud, el sexo, la edad, la pigmentación de la piel y el tipo de técnica utilizada para medir la vitamina D, encontrándose 394 estudios que incluían 33.266 sujetos, con un valor promedio de 25(OH)D de $54 \mathrm{~nm} / \mathrm{l}$, siendo para las mujeres de $56 \mathrm{~nm} / \mathrm{l}$ y para los hombres de $50 \mathrm{~nm} / \mathrm{l}$. No se encontró relación aparente entre estos niveles y la pigmentación cutánea, la latitud sobre el nivel del mar, ni la técnica utilizada.

Los análisis llevados en Bogotá en dos laboratorios privados con un número de muestras importante (2.256 pacientes entre noviembre del 2012 y abril del 2013) mostraron suficiencia en su concentración en solo $30 \%$ de los pacientes ${ }^{(7-8)}$.

En ese mismo evento se llevó a cabo una encuesta en la Asociación Colombiana de Endocrinología, la cual fue respondida por un $50 \%$ de sus miembros, mostrando interés en su dosificación en 93\% de las encuestas, especialmente para las pacientes mayores de 65 años, teniendo como dificultad para hacerlo el poder contar con laboratorios competentes, además mostró su interés por establecer protocolos de tratamiento en estos pacientes $^{(9)}$.

El II Consenso Colombiano para el Manejo de la Osteoporosis Postmenopáusica, llevado a cabo por un grupo importante 
de expertos y publicado en la Revista Colombiana de Reumatología, en el año 2018, recomendó en el caso de suplementación 1000 UI/día, en el caso de insuficiencia 1000-2.000 UI/día y en el caso de deficiencia 2.000-6.000 UI/día ${ }^{(10)}$.

La conferencia internacional "Controversias en vitamina D” llevada a cabo en Pisa, Italia, en junio del 2017, revisó y analizó la literatura científica internacional en relación con el papel que juega la vitamina $\mathrm{D}$ en la salud humana ${ }^{(11)}$.

Mediante un panel en el cual participaron expertos internacionales se discutieron y aprobaron los siguientes tópicos: 1) Pruebas para dosificar la vitamina $D$ y definición de hipovitaminosis, 2) Efectos esqueléticos y extraesqueléticos de la vitamina D y, 3) Uso terapéutico de la vitamina D.

En relación con el primer ítem, las pruebas de laboratorio utilizadas para determinar la concentración de 25-hidroxivitamina D se define como la suma de los niveles de vitamina D3 y vitamina D2, los otros metabolitos son usados en investigación especialmente y no en situaciones clínicas.

Su papel en el origen del raquitismo y la osteomalacia está claramente establecido, además juega un papel importante en la génesis de otro tipo de patologías como son alteraciones dermatológicas, cardiovasculares, inmunes y metabólicas. La piel representa un claro ejemplo de acción no esquelética de la vitamina D. Su papel en el sistema inmune fue propuesto hace ya varias décadas cuando el receptor de la vitamina D mostró que podría ser expresado en los linfocitos T. Además, se ha propuesto que sus niveles bajos podrían llevar a un aumento del riesgo de infecciones, inhibiendo el sistema adaptativo de respuesta inmune, dando como resultado ciertas patologías como esclerosis múltiple y diabetes tipo 1; pero estas observaciones no han sido demostradas por estudios aleatorizados con un número suficiente de pacientes. Sin embargo, una de las observaciones más consistentes ha sido su relación con la obesidad y la diabetes tipo 2 .

En relación con su uso terapéutico, este panel de expertos determinó, mediante el estudio de varios metanálisis, la eficacia de la vitamina D3 en mejorar los niveles de la 25-hidroxivitamina D, pero tanto la vitamina D2 como la D3 pudieran ser utilizadas igualmente en el tratamiento y prevención de su hipovitaminosis.

En el presente número de nuestra revista se publica el artículo Tuberculosis y vitamina D: una relación intrigante, escrito por Ramirez-Ramos y colaboradores, en el cual se presenta una revisión detallada de los estudios que explican el papel de la vitamina $\mathrm{D}$ en la susceptibilidad para el desarrollo de la tuberculosis y los resultados de los estudios clínicos de suplencia de la misma publicados hasta la fecha.

El papel del sol (vitamina D?) en el tratamiento de la tuberculosis ha sido descrito en la literatura internacional desde la época del escritor alemán Thomas Mann en su obra $L a$ montaña mágica ${ }^{(12)}$, en la cual describe un sitio en las montañas suizas adonde se enviaban los pacientes con tuberculosis para que mejoraran de sus dolencias mediante la exposición al sol, también en Bogotá se llevó a cabo una copia posiblemente del anterior, el Hospital San Carlos, el cual está dotado de un hermoso bosque adonde sacaban a asolear a los pacientes. ¿Será que hasta ahora estamos empezando a entender la gran bondad de los rayos solares?

\section{Enrique Ardila \\ Editor}

Revista Colombiana de Endocrinología, Diabetes y Metabolismo

Referencias

1. Jácome A. Calciferoles: Las hormonas del momento. En Historia de la vitamina D. Iglesias A., Restrepo JF.,Toro CE. pp. XIII-XXVI. Artes Gráficas (editorial), Barranquilla, junio 2008.

2. Hormaza MP., Cuesta D., Martínez LM., Massaro MM., Campo MN., Vélez MP. et al. Niveles séricos de 25 hidroxivitamina D en mujeres no menopáusicas, menopáusicas y postmenopáusicas. Rev. Colomb. Obstet. Ginecol. 2011. 62: 231-236.

3. Vásquez NH, Pinzón A., Santofimio D. Niveles de vitamina D en pacientes con osteoporosis en el Hospital Universitario Hernando Moncaleano Perdomo de Neiva. Trabajo de grado Facultad de Salud, Universidad Surcolombiana 2013.

4. Molina JF., Molina J., Escobar JA., Betancur JF., Giraldo D. Niveles de 25 hidroxivitamina D y su correlación clínica con diferentes variables metabólicas en una población de mujeres postmenopáusicas. Acta Me. Colomb. 2011.36.1823.

5. González D., Zuñiga C., Kattah W- Insuficiencia de vitamina D en pacientes adultos con baja masa ósea y osteoporosis en la Fundación Santafé de Bogotá, 20082009. Rev. Col.Reumatol. Dic 2010.17849:212-218.

6. Rosero 0. Vitamina D y salud ósea en la mujer postmenopáusica. Revisión. Rev. Col. Endocrinol. Diabet. Metabol. Feb 2015. Vol 2 (1): 14-19.

7. Escandón de C. Laboratorio Analizar 2014. Informe.

8. Laverde G. Laboratorio de Investigaciones hormonales 2014. Informe.

9. Ardila E. XXIII Congreso Colombiano de Medicina Interna. Cartagena 2014.

10. Medina A., Rosero O., Rueda PN., Sánchez F., Chalem M., González MA. et al. II Consenso colombiano para el manejo de la osteoporosis postmenopáusica. Rev. Colomb. Reumatol. 2018. 25(3):184-210.

11. Giustina A., Adler RA.,Binkley N., Bouillon R., Ebeling PR., Lazaretti- Castro M., et al. Controversies in Vitamin D : Summary Statement From an International Conference. J Clin Endocrinol Metab. 2019. 104: 234-240.

12. Mann T. La montaña mágica. 1964. pp. 7-844. Editorial Diana México DF. 